\title{
MS29-04 | Hydrogen Positions in Small Organic Molecules Determined by 3D ELECTRON DifFraction
}

Lightowler, Molly (Stockholm University, Stockholm, SWE)

Understanding the distribution of hydrogen atoms is an essential part of any crystal structure analysis. However, the localization of hydrogen atoms is often hindered by their small scattering power. The vast majority of structures are identified through X-ray crystallography, yet this technique is limited to micrometre-sized crystals and since $\mathrm{X}$-rays only interact weakly with matter, acquiring a strong-enough signal to unveil fine structural details, such as hydrogen positions, is often impossible. 3D electron diffraction has shown to be powerful in structure determination of nano- and micron-sized organic crystals. Hydrogen positions in nanocrystals could be revealed as a result of the stronger interaction of electrons with matter and the use of dynamical refinement. We report the structures and direct localization of hydrogen atoms in nanocrystalline, small organic molecules, using 3D electron diffraction and only kinematic approximation. Hydrogen positions were found in structures from three individual molecules (B-glycine, sucrose and ABTC). Every hydrogen position was determined within the structure of $B$ glycine, whilst only certain hydrogen positions were found in sucrose and ABTC. The results demonstrate that the ability to locate hydrogens depends upon their environment within the structure. The flexible hydrogens, with varying positions averaging out over the crystal, cannot be determined, whilst the rigid hydrogens, e.g. those that are hydrogen bonded or aryl hydrogens, are easier to locate. The results demonstrate that 3D electron diffraction can achieve determination of fine structural details from single crystals of small organic molecules with nanosized dimensions. 